\section{Larynx- und Hypopharynxkarzinom: Langzeitergebnisse zum Larynxerhalt}

\begin{abstract}
In der Studie EORTC 24954 wurden die sequenzielle und die alternierende Radiochemotherapie bei Patienten mit lokal fortgeschrittenem Larynx- und Hypopharynxkarzinom über einen Zeitraum von 10 Jahren verglichen.
\end{abstract}

D ie zeitgleiche und die sequenzielle Radiochemotherapie haben sich als effektive Therapie bei Patienten mit lokal fortgeschrittenem Plattenepithelkarzinom des Larynx oder Hypopharynx erwiesen. Zur Frage des optimalen Vorgehens zum Larynxerhalt wurden in der EORTC-Studie 24954 bei 450 Patienten mit resezierbarem Larynx- oder Hypopharynxkarzinom die sequenzielle und die alternierende Radiochemotherapie randomisiert miteinander verglichen. Nach einer medianen Beobachtungsdauer von 6,5 Jahren waren das Überleben mit einem funktionsfähigen Larynx, das progressionsfreie Überleben und das Gesamtüberleben in beiden Gruppen vergleichbar. Nun wurden Daten mit ei- ner Beobachtungsdauer von 10,2 Jahren publiziert. Im sequenziellen Arm erhielten die Patienten eine Induktionstherapie mit Cisplatin und 5-Fluorouracil (5-FU), anschließend Bestrahlung mit 70 Gy bei Ansprechen und eine totale Laryngektomie und postoperative Bestrahlung bei Nichtansprechen; im alternierenden Arm Cisplatin und 5-FU abwechselnd mit 3 zweiwöchigen Radiotherapie-Zyklen mit je $20 \mathrm{~Gy}$.

Das 10-Jahres-Überleben mit einem funktionsfähigen Larynx (primärer Endpunkt) war in beiden Armen vergleichbar $(18,7 \%$ bei sequenzieller Therapie vs. $18,3 \%$ bei alternierender Therapie), ebenso das Gesamtüberleben $(33,6$ vs. $31,6 \%$ ). Bei sequenzieller Therapie wurden allerdings mehr Todesfälle aufgrund von Toxizitäten und Infektionen beobachtet (12 vs. $4,5 \%$ ), dagegen waren kardiovaskulär bedingte Todesfälle bei alternierender Therapie häufiger ( $4,7 \mathrm{vs.}$ $10,9 \%$ ). Die Rate an späten Toxizitäten war in beiden Armen vergleichbar.

Auch die Häufigkeit lokoregionärer Rezidive war in beiden Armen vergleichbar. In der Gruppe mit alternierender Therapie gab es einen Trend zu mehr Fernmetastasen und zweiten Primärtumoren, aber auch einen Trend zu einer höheren Rate beim Larynxerhalt.

Fazit: Diese 10-Jahres-Daten bestätigen frühere Ergebnisse, die ebenfalls eine vergleichbare Effektivität von sequenzieller und alternierender Radiochemotherapie bei Patienten mit lokal fortgeschrittenem Larynx- und Hypopharynxkarzinom gezeigt hatten.

Judith Neumaier

De Figueiredo BH et al. Long-term update of the 24954 EORTC phase III trial on larynx preservation. Eur J Cancer. 2016;65:109-12.

\title{
Schilddrüsenkarzinom: Wie viele Lymphknoten untersuchen?
}

Eine aktuelle Studie liefert erstmals eine empirisch basierte Risikoabschätzung für einen nach der Operation unerkannten Lymphknotenbefall bei Patienten mit papillärem Schilddrüsenkarzinom.

$\mathrm{M}$ an geht davon aus, dass bei Patienten mit papillärem Schilddrüsenkarzinom postoperativ ein Risiko besteht, Lymphknotenmetastasen zu übersehen, wenn die Lymphknoten nur eingeschränkt untersucht wurden. Die Höhe des Risikos wurde bisher nicht objektiv quantifiziert, könnte aber nachfolgendes Management und Kontrolluntersuchungen beeinflussen.

In einer Studie wurden nun 78.724 Fälle von Patienten mit lokalisiertem, $\geq 1 \mathrm{~cm}$ großem papillärem Schilddrüsenkarzinom und einem oder mehreren untersuchten Lymphknoten nach Thyreoidektomie analysiert, die von 1998 bis 2012 in der US-amerikanischen Krebsdatenbank registriert worden waren. $38.653(49,1 \%)$ von ihnen waren nodal positiv und wurden zur Risikoabschätzung falsch-negativer Ergebnisse herangezogen. Die Wahrscheinlichkeit, mit der Patienten mit befallenen Lymphknoten fälschlicherweise als nodal negativ diagnostiziert wurden, lag bei $53 \%$, wenn nur ein einziger Lymphknoten untersucht wurde, und sank auf unter $10 \%$, wenn > 6 Lymphknoten untersucht wurden. Anschließend wurde die tatsächliche Prävalenz positiver Lymphknoten in der gesamten Studienpopulation in den Stadien T1b-4 mit 36,9-78,6\% berechnet; nach Berücksichtigung falschnegativer Befunde war sie deutlich höher (47,1-98,4\%).

Zuletzt wurde errechnet, wie viele Lymphknoten man untersuchen muss, um einen übersehenen Lymphknotenbe- fall mit $90 \%$ Sicherheit auszuschließen: Bei Patienten mit einer T1b-Erkrankung waren das 6, bei Patienten mit einer T2Erkrankung 9 und bei denen mit einer T3-Erkrankung 18 Lymphknoten. Sensitivitätsanalysen ergaben, dass 3,4 bzw. 8 Lymphknoten untersucht werden müssen, um eine vergleichbar verlässliche Beurteilung des Nodalstatus zu erzielen.

Fazit: Das Risiko für einen nicht erkannten Lymphknotenbefall ist bei Patienten mit papillärem Schilddrüsenkarzinom postoperativ vom Stadium des Primärtumors und der Anzahl untersuchter Lymphknoten abhängig. Chirurgen und Pathologen könnten diese Risikoabschätzung verwenden, um etwa das Lymphknotenfeld für eine Bestrahlung zuverlässig abzuschätzen.

Judith Neumaier

Robinson TJ et al. How Many Lymph Nodes Are Enough? Assessing the Adequacy of Lymph Node Yield for Papillary Thyroid Cancer. J Clin Oncol. 2016;34(28):3434-9. 